
cultures

Les cahiers de l'Acedle

15-1 | 2018

La conceptualisation grammaticale

\title{
La conceptualisation grammaticale : entre grammaire artificielle et grammaire mentale
}

Henri Portine

\section{(2) OpenEdition}

1 Journals

Édition électronique

URL : http://journals.openedition.org/rdlc/2662

DOI : $10.4000 /$ rdlc.2662

ISSN : 1958-5772

Éditeur

ACEDLE

Référence électronique

Henri Portine, « La conceptualisation grammaticale : entre grammaire artificielle et grammaire mentale », Recherches en didactique des langues et des cultures [En ligne], 15-1 | 2018, mis en ligne le 02 janvier 2018, consulté le 21 décembre 2020. URL : http://journals.openedition.org/rdlc/2662 ; DOI : https://doi.org/10.4000/rdlc.2662

Ce document a été généré automatiquement le 21 décembre 2020.

\section{(1) () ()}

Recherches en didactique des langues et des cultures is licensed under a Creative Commons AttributionNonCommercial-NoDerivatives 4.0 International License 


\title{
La conceptualisation grammaticale : entre grammaire artificielle et grammaire mentale
}

\author{
Henri Portine
}

\section{Mise au point terminologique sur « grammaire »}

1 Le processus de "conceptualisation grammaticale» met en correspondance "de la grammaire » et des «processus mentaux » (des concepts, des relations entre concepts et les manifestations empiriques de ces concepts). Nous nous interrogerons donc d'abord sur la notion de grammaire ${ }^{1}$.

2 Dans cet article $^{2}$, nous emploierons les termes de "grammaire artificielle» (formule empruntée à Stanislas Dehaene, cf. son séminaire de 2016-2017 au Collège de France) et "grammaire mentale», ce qui n'est pas l'usage général. D'une part, ce qui est appelé « grammaire artificielle » est souvent considéré comme « la » grammaire, mais désigne les grammaires papier ou numériques conçues par des grammairiens et qui sont des constructions hypothétiques. D'autre part, on parle généralement de "grammaire intériorisée ", là où nous employons "grammaire mentale ». Nous évitons "grammaire intériorisée » : elle signifie souvent « grammaire construite par le sujet en intégrant des règles - et non des données - fournies de l'extérieur ». Dans ce sens, "grammaire intériorisée » nous semble proprement inintéressant. Nous nous situerons à un niveau intermédiaire entre grammaires artificielles et grammaire mentale, parce que nous considérerons que c'est le niveau d'opérativité de la didactique de la grammaire : on vise la construction / transformation de la grammaire mentale de l'apprenant en l'aidant à élaborer ses propres règles et ses propres représentations (il y a enseignement de la grammaire lorsqu'on ne vise que l'apprentissage de règles externes sans qu'il y ait travail de ré-analyse par l'apprenant).

3 Traditionnellement, le palier supérieur du dispositif grammatical est la phrase (cf. Seguin, 1993). Tesnière a donné la métaphore de la phrase petit drame $(1959: 102, \S 48):<$ des 
actants sujet et compléments du verbe - verbe - circonstants adverbiaux ou sous la forme de compléments > correspondent à < acteur(s) - action - décors >. La grammaire vise donc la structuration phrastique, mais elle a aussi deux autres fonctions parce que l'emploi de telle forme dialectale ou sociolectale ou le choix (qui peut être infra-conscient) de telle marque (connecteur, temps verbal, etc.) ont des incidences d'une part sur le repérage socio-dialectal du locuteur et d'autre part sur la stratégie discursive employée (cf. figure 1).

Figure 1 - Fonctionnalités de la grammaire

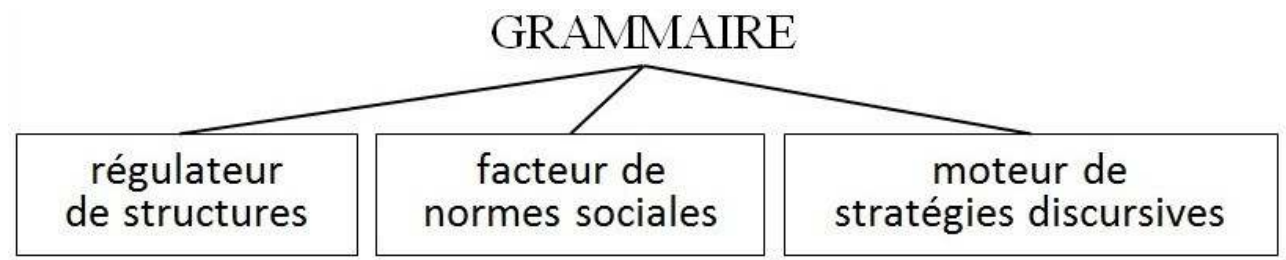

\section{Deux conceptions de la conceptualisation grammaticale}

Besse définit ainsi la conceptualisation grammaticale $(1974: 38)$ :

[la conceptualisation c'est] une attitude réflexive sur [la] pratique du français, l'objectif étant de se forger une conceptualisation, une sorte de règle, qui n'a rien d'absolu, mais qui a une valeur opératoire et qui peut porter sur des microsystèmes.

Besse compare cette notion de conceptualisation (que nous nommerons " conceptualisation-réarrangement ») à la grammaire inductive (1974:39), mais, ajoute-til, (ibid., 43) ici l'exercice ne vise pas à apprendre une règle ou une loi de la langue, mais à saisir le fonctionnement de certains énoncés de cette langue. Cette saisie intuitive, mal exprimée, nous paraît plus importante qu'une formulation précise.

Janine Courtillon-Leclerq (1974) complète ce panorama en montrant comment les paraphrases jouent un rôle important dans cette démarche de conceptualisation. Elle utilise une "technique paraphrastique" pour faire ressortir les valeurs d'éléments utilisés comme pivots (les valeurs de « voilà » et les différents sens de " penser »).

Dans Besse \& Porquier (1984: 113-115), la présentation de la démarche de conceptualisation (section Perception métalinguistique de l'apprenant) n'est pas sensiblement modifiée, mais on y trouve deux nouveaux points : l'aspect métalinguistique des opérations est souligné; la notion de corpus (non présélectionné par le professeur mais issu d'un recueil d'erreurs) apparaît. Remarquons tout d'abord que cette notion de conceptualisation se positionnait en rupture avec la méthodologie structuro-globale audio-visuelle et s'inscrivait dans le contexte d'apparition des différents courants de prise en compte de l'erreur (interlangue ${ }^{3}$, pédagogie de la faute d'André Lamy au BELC, etc.). Faisons maintenant quelques remarques sur cette notion:

- elle a été conçue pour le « niveau 2 » à l'époque de la division entre Français fondamental 1 et Français fondamental 2; ce n'est donc pas une méthodologie pour construire une première représentation des faits grammaticaux ;

- elle suppose une vision modulaire de la grammaire mentale de l'apprenant (elle se construit par apports spécifiques de formes et d'effets, successifs mais non linéaires), vision assez 
partagée aujourd'hui mais toute modularité entraîne une question: quelle architecture permet d'associer les modules au sein d'un système global ?

- elle comporte de légères contradictions (elle est le produit de réflexions empiriques) : on se forge une règle mais on n'apprend pas cette règle (qu'en fait-on ?) ; on part des productions des élèves mais on admet aussi des exemples issus de dialogues; toutefois ces légères contradictions n'empêchent pas une vision d'ensemble cohérente qui évoque certains ateliers d'écriture ou certaines pratiques Freinet;

- Besse semble avoir une confiance absolue en l'intuition des apprenants mais considère que les apprenants, en général, formulent des règles qui sont des réactualisations de règles qu'ils ont apprises et oubliées (Besse \& Porquier, 1984: 115), ce qui semble contradictoire avec la conception d'ensemble ;

- si Courtillon-Leclerq (1974) conjoint conceptualisation et paraphrase, Besse n'aborde pas ce point; dans Besse \& Porquier (1984) conceptualisation et reformulation sont totalement dissociées, en revanche, reformulation et paraphrase sont liées (Besse \& Porquier, 1984 : 138-145) mais il s'agit des paraphrases de "la nouvelle méthodologie DVV (De Vive Voix)» proposée par Argaud et Marin (cf. Portine, 2010 : 124) au début des années 1970 ;

- elle recourt à un terme dans un sens non canonique ; Courtillon-Leclerq (1974: 45, note) le remarque : ce terme [conceptualisation] prête à ambiguïté et heurte le psychologue qui lui donne une toute autre acception.

8 Ce qui précède pourrait être considéré comme de l'archéologie, mais la didactique de la grammaire n'ayant pas fait de très grands progrès entre les années 1970 et aujourd'hui (2017), nous allons continuer cette investigation. Il se pourrait que la situation change grâce à l'évolution de la neuropsychologie .

9 Si la notion de conceptualisation-réarrangement apparaît dans le cadre du CREDIF (1959-1996), une autre notion de conceptualisation s'inscrit dans un cadre très différent, provincial (chose peu fréquente, à l'époque), celui du CLAB (Centre de linguistique appliquée de Besançon, fondé au début des années 1960); nous l'appellerons «conceptualisation-construction». Au début des années 1970, des enseignant(e)s décident de fonder leur propre méthode sur des principes s'inspirant des «événements de Mai 68 »; ce sera C'est le printemps. La méthode paraît en 1976. En se plaçant dans la suite d'un courant de pensée tel que celui de Mai 68 , on ne saurait imposer des règles. L'idée sera donc la suivante : comment aider des apprenants à se construire leurs propres règles grammaticales tout en leur offrant un matériel (corpus ou un mini-corpus) qui leur permette de dégager (à la fois collectivement et individuellement) leurs propres règles sans pour autant sombrer dans des représentations fantaisistes ou totalement inadéquates?

10 Ce qui va suivre s'appuie sur une bande vidéo (alors des galettes analogiques) enregistrée par Christian Lavenne (l'un des auteurs de C'est le printemps) au CLAB, bande vidéo malheureusement disparue (il faut donc entrer ici dans le témoignage ${ }^{5}$ ). Cette bande vidéo portait sur un corpus de 12 mini-dialogues (Montredon et alii, 1982:101) destiné à faire conceptualiser, par les apprenants, les emplois de en pronom (mais la proposition pédagogique donnée dans le Livre du professeur, page 107, ne correspond pas à la bande vidéo et reste assez traditionnelle). La séquence des mini-dialogues proposés aux apprenants est préenregistrée et n'est pas ordonnée. Les points suivants ont frappé l'auteur de ces lignes:

a. dans l'élucidation des premiers mini-dialogues, en n'est pas entendu par les apprenants (on constate aisément que nombre de locuteurs allophones en français ne disent pas «j'en 
veux » mais «je veux » sans que cela trouble leurs échanges); Christian Lavenne ne relève pas cette absence et ce n'est qu'après l'exploitation des premiers mini-dialogues que en commence à être entendu par certains puis cette perception se généralise, ce qui permet de revenir sur l'écoute des premiers dialogues ;

b. lorsque commence à s'élaborer une règle collective, le retour sur les mini-dialogues (pour lesquels en n'a pas été élucidé ni même parfois perçu) permet d'organiser un premier test de vérification; il y a donc confrontation de la donnée en train d'être analysée aux données fournies par les mini-dialogues précédents (réseau de données) ;

c. lorsqu'une règle ou une amorce de règle a été élaborée, elle est soumise pas à pas aux minidialogues suivants afin de la conforter ou de l'ajuster ou encore de la remettre totalement en cause ; il s'agit donc ici d'un processus d'évaluation à la fois individuelle et collective ;

d. certains mini-dialogues comportent les pronoms le, la (construction directe de l'objet) au lieu du pronom en; ils permettent une analyse contrastive ;

e. un groupe-classe n'est pas homogène (Besse l'avait déjà remarqué, cf. 1974) et certains apprenants vont s'appuyer sur l' " expertise partielle » d'autres apprenants qui, en retour, bénéficieront des difficultés des premiers, ce qui les obligent à affiner leurs points de vue (le fait d'être amené à expliciter son point de vue est facteur de conceptualisation) ;

f. toute conclusion est partielle, dans l'attente de confirmations ou d'infirmations.

11 À chacune de ces remarques, on peut associer un commentaire :

a. on a tendance à distinguer radicalement le niveau de la perception et le niveau de la compréhension en renvoyant le niveau de la perception à un problème de phonétique; le point (a) nous fait penser que la perception doit aussi être traitée sur le plan cognitif ; on ne peut chercher à concevoir les contours d'une question que lorsqu'on perçoit la manifestation empirique de cette questio

b. une maîtrise de l'élucidation du «non-encore-connu » passe par la mise en réseau (cf. d' cidessous) de l'élément à élucider en contrastant certains usages (ici le mini-dialogue sur lequel on travaille et les mini-dialogues précédents) ;

c. notre appareillage neurocognitif fonctionne de façon partiellement aléatoire (c'est aussi l'un des points discutés actuellement en neuropsychologie) ; il est donc important d'apprendre à s'autoévaluer pour ajuster non seulement son dire mais aussi ses représentations langagières ; ces procédures d'autoévaluation et d'ajustement supposent le développement d'une véritable plasticité mentale qui a existé dans l'enfance et dans l'adolescence de tout locuteur mais qui peut avoir été perdue ;

d. la contrastivité des constructions en le, la et en en permet de mettre en évidence un type de réseau; il y a encore peu de réflexions sur la notion de réseau : si je change tel élément en tel point de la chaîne, quelles en sont les conséquences du point de vue syntagmatique proche (effets constructionnels) et lointain (effets sur la stratégie discursive) ?;

e. tout apprentissage, y compris linguistique, est social ; cette dimension sociale doit donc être instaurée aussi dans le « cours de grammaire $»^{6}$;

f. c'est sans doute là le point le plus délicat parce qu'un apprenant aime bien savoir s'il a compris ou non ce dont il était question; il faut alors apprendre que toute pratique linguistique est instable.

12 On trouve une procédure analogue de conceptualisation sur un site web réalisé à l'Université d'Alep ${ }^{7}$ par une équipe d'enseignants vraisemblablement sous la direction de Zoubeida Kadi. Cette procédure se déroule en six étapes : (a) observation du phénomène, (b) manipulation des énoncés et formulation d'hypothèses, (c) vérification des hypothèses, (d) formulation de lois, de régularités ou de règles et établissement de procédures, (e) phase d'exercisation, (f) réinvestissement contrôlé. 
13 On trouvera aussi une autre manifestation de cette forme de conceptualisation grammaticale, portant sur les temps verbaux en français, dans Montredon (1987) : les apprenants sont amenés à réfléchir sur leur propre pratique d'apprentissage pour conceptualiser leurs emplois des temps du passé.

\section{Une troisième forme de conceptualisation grammaticale?}

Nous avons vu que les deux conceptions précédentes s'appelaient conceptualisation, mais sans que la notion de concept soit directement présente ${ }^{8}$. Il en est ainsi parce que la focalisation se fait sur la notion de règle. D'un certain point de vue, cela est tout à fait légitime : les règles sont des maximes d'action. Elles permettent donc au sujet apprenant de guider sa propre production dans son rôle de locuteur ou de scripteur mais aussi sa reconnaissance (ou compréhension) dans son rôle d'interlocuteur ou de lecteur. La force des règles, c'est de déboucher sur des résultats, d'avoir une certaine technicité ; leur faiblesse, c'est d'avoir un pouvoir d'action limité et de porter le risque d'exceptions. En revanche, la force des concepts, c'est de pouvoir subsumer des situations différentes et des cas diversifiés; leur faiblesse, c'est leur abstraction. Si les règles sont des outils opératoires, les concepts sont des outils de compréhension.

Il y a bien, cependant, sous les emplois grammaticaux, des concepts à l'œuvre. Prenons un exemple : la notion de durée souvent associée à l'imparfait ne tient pas longtemps si l'on prend le terme sous son aspect empirique : ce qui dure se déroule sur une longue période ${ }^{9}$ . En revanche, si l'on prend le terme durée sous l'angle d'un point de vue, c'est-à-dire comme renvoyant à "procès saisi dans sa durée", il n'est toujours pas totalement adéquat mais il devient beaucoup moins critiquable.

\section{Conceptualisation et heuristiques}

16 On peut opposer le recours à des dialogues (Miquel, 2013) et le recours à des fragments (Abry \& Chalaron, 2015) :

- des fragments se focalisent sur le point à traiter mais en étant moins contextualisés ;

- des dialogues inscrivent clairement les marques grammaticales dans des intentions signifiantes et des stratégies discursives mais peuvent diluer le point à traiter.

Élargir le plan de l'expression permet de mieux jouer sur le rapport entre le plan purement grammatical (les opérations de bas niveau) et le plan du discours (les opérations de haut niveau). Cette opposition, faite depuis les années 1970 (sur un plan plus général : perceptif notamment), illustre les démarches de compréhension (cf. figure $\left.2^{10}\right)$. 
Figure 2 - La complémentarité Haut-niveau / Bas-niveau

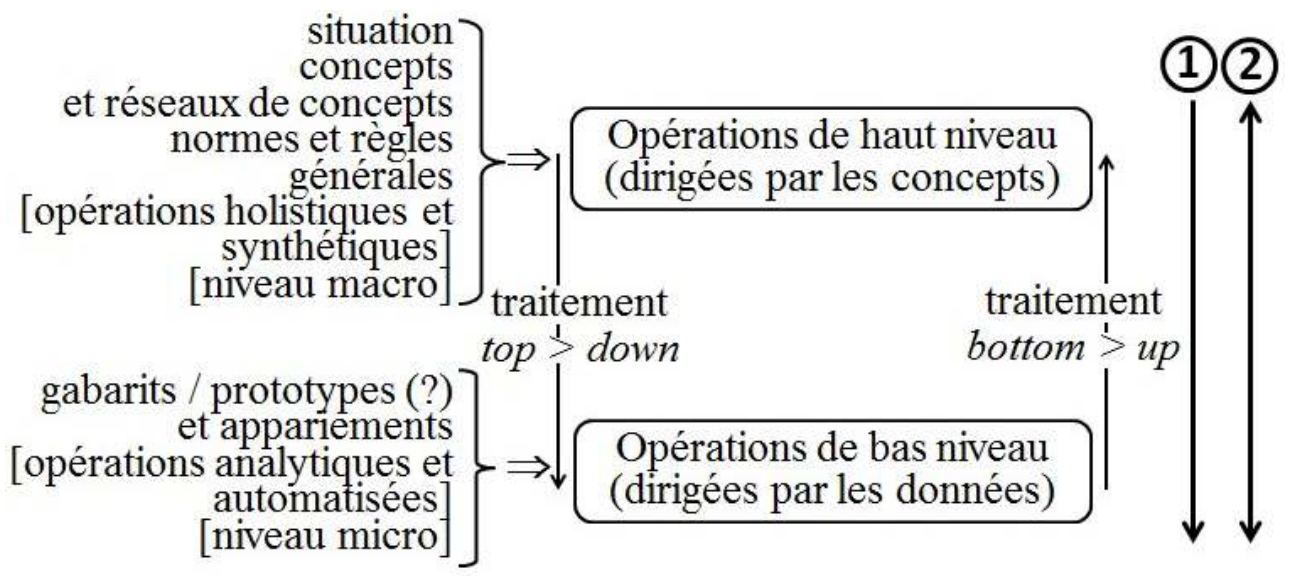

Repérer telle marque grammaticale dans un texte ou dans un dialogue, c'est pouvoir lui donner une valeur d'emploi. Mais cette démarche a une limite: plus on commente la valeur sémantique d'une marque grammaticale, plus on l'individualise et, donc, moins elle permet une caractérisation générale de cette marque grammaticale. Tout(e) enseignant(e) a eu cette impression de ne pouvoir atteindre la réalité profonde de l'explication d'un emploi d'une marque grammaticale donnée, quel que soit son niveau de formation en linguistique: nous nous heurtons ici à un phénomène que l'on peut considérer comme ayant une part d'aléatoire. C'est pourquoi nous allons considérer les corpus d'observation comme des heuristiques.

19 L'emploi d'« heuristique » en didactique de la grammaire n'est pas nouveau (cf. Cuq, $1996: 41,45,50,104)$, mais toujours en position adjectivale et toujours situé du côté de l'apprenant (ce sur quoi l'enseignant(e) n'a qu'une prise indirecte), alors que nous visons « un partage ». De plus, ces emplois d'« heuristique» concernent la constitution d'une conscience métalinguistique.

Avant de préciser ce que nous entendrons ici par «heuristique» (qui se situe dans le prolongement de Aguerre, 2010), il convient de distinguer "activité épilinguistique » et "activité métalinguistique». Le terme épilinguistique a été introduit, sans véritable définition, par Culioli ${ }^{11}$ (1968: 108) : « le langage est une activité qui suppose, elle-même une perpétuelle activité épilinguistique (définie comme "activité métalinguistique non consciente»)». Remplaçons activité métalinguistique par activité de rétro-contrôle; remplaçons ensuite non-conscient par infra-conscient ou peu conscientisé. On aboutit à une nouvelle définition: "l'activité épilinguistique est une activité d'auto-réflexion ou de rétro-contrôle par le locuteur sur sa propre production ", et ajoutons la fonction de cette activité : "afin de l'ajuster à sa représentation de la correction linguistique ou de sa stratégie discursive en cours ${ }^{12}$. À l'opposé, une activité métalinguistique est une activité consciente fondée sur un raisonnement (pré)scientifique. Une capacité à avoir une activité épilinguistique en français est donc un but et non un moyen, alors qu'une activité métalinguistique sera un moyen pour atteindre des buts divers (description scientifique, amélioration de son activité épilinguistique, etc.).

21 Nous appellerons "heuristique» le dispositif mis en place par l'enseignant(e) pour favoriser, guider le processus de résolution de problème ayant pour but la constitution d'un concept ou d'une règle. Cette heuristique (remarquons qu'il s'agit là d'un nom), nous la situons d'abord du côté de l'enseignant(e) et seulement ensuite du côté de 
l'apprenant pour une raison très simple: on peut postuler des processus heuristiques chez l'apprenant mais, s'ils existent, on ne peut en connaitre le mécanisme que par des témoignages indirects.

Nous empruntons à la psychologie ce passage d'«heuristique » du statut adjectival au statut nominal :

Traditionnellement en psychologie, notamment dans son acception française (jusqu'au milieu des années 1970), le terme heuristique s'employait comme adjectif pour qualifier une démarche, un procédé, une information, etc. qui enrichit les connaissances et qui aide à la découverte de solutions nouvelles. [...] une évolution sémantique s'est dessinée. Le terme heuristique est maintenant utilisé comme substantif pour désigner une méthode rapide et simple de traitement permettant de trouver des réponses aux problèmes d'évaluation et de jugement. (Cadet \& Chasseigne, 2009:179-180)

On aura (a) une situation S à traiter, (b) une heuristique H qui apportera des informations susceptibles d'être applicables, (c) l'attente d'un résultat C qui consiste dans le choix d'une valeur conceptuelle ou d'une modalité d'action. Dans le cadre d'une didactique de la grammaire, l'enseignant(e) propose la situation S (corpus ou autre), guide les apprenants en recourant à une heuristique $\mathrm{H}$ partagée et les aide à évaluer le résultat $\mathrm{C}$ obtenu et à le considérer comme provisoire.

La base du travail pour l'apprenant est donc un travail de repérage (que l'on trouve aussi en français langue maternelle, cf. Wilmet, 2016: 134). Si nous représentons le travail grammatical (dans des séances dédiées ou au fil des apprentissages de la scripturalité et de l'oralité) comme l'emboîtement de trois types d'activités (figure 3), une heuristique mobilisera le travail de description et d'explicitation et le travail de rétro-contrôle.

Figure 3 - Fonctionnalités du travail grammatical

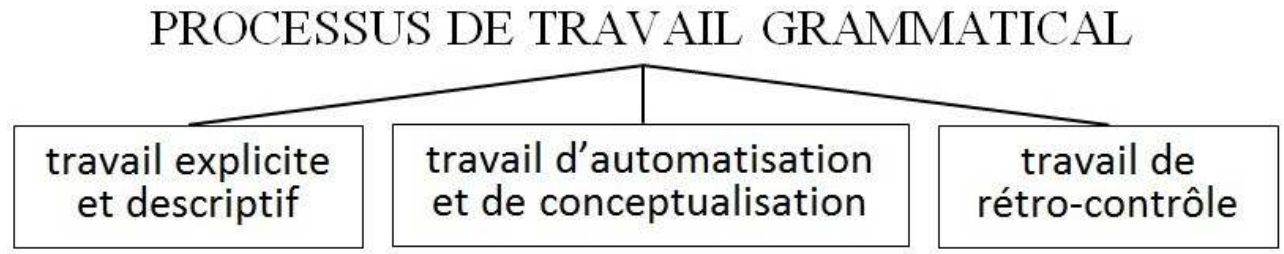

Une heuristique opère au niveau de ce que nous avons appelé « palier intermédiaire entre les grammaires artificielles et la grammaire mentale ». Il est banal de rappeler qu'un(e) enseignant(e) ne peut se référer qu'aux grammaires artificielles puisque nous n'avons pas d'accès direct aux mécanismes de notre grammaire mentale.

Il est toujours difficile de comparer des approches didactiques. Nous serons donc prudent. Il nous semble cependant qu'il existe des affinités entre les orientations proposées ici et celles de l'équipe (angliciste) Charlirelle (de l'Institut Charles V de l'Université Paris 7 Diderot) et plus précisément du GEPED (Groupe d'Etudes en Psycholinguistique et Didactique) qui a été (co-)fondé par Danielle Bailly.

\section{Un exemple d'heuristique}

Il est impossible de détailler le travail fait dans une classe de langue. La présentation qui suit, forcément schématique, portera sur l'abord (donc sur un traitement non exhaustif) 
du pronom relatif dit «simple» (non composé) dans le cadre de la proposition relative adjointe.

En français, la proposition relative appartient au domaine des subordonnées (TrévisiolOkamura \& Kaheraoui, 2015) et a une structure très précise qui est de type «pronom relatif + reste de la proposition » (le pronom relatif appartient à la proposition relative alors que, pour les autres subordonnées, on a « conjonction + proposition " parce que la conjonction articule la proposition à la «principale»). Nous éliminons les types complexes (exemple emprunté à Ferdinand Brunot: l'homme à l'enterrement duquel je suis allé... où à l'enterrement appartient à la proposition relative bien que situé avant duquel). La didactique de la grammaire hésite entre l'abord de la problématique par le pronom relatif ou par la proposition relative. Mettre en avant le pronom relatif ${ }^{13}$, c'est privilégier la relation anaphorique alors que la focalisation sur la proposition relative ${ }^{14}$ revient à mettre l'accent sur l'opération de détermination (pour le type adjoint). Certes, les deux propriétés (anaphoricité et détermination) vont se retrouver dans les deux guidages proposés par l'enseignant(e) mais l'un étant souvent occulté.

Nous ne pouvons développer l'exposition d'un dispositif-processus (dispositif dans sa forme, processus dans sa réalisation) qui sera variable selon le niveau des apprenants, leurs langues d'origine et leur degré d'activité et de collaboration à une réflexion collective, mais nous pouvons caractériser le dispositif à la base du processus sous la forme de quelques points :

- le matériel de départ est constitué de mini-récits, de mini-dialogues ou de miniargumentations, mais chaque composante doit avoir son autonomie et permettre soit de mettre en jeu une intention signifiante particulière, soit d'illustrer clairement la ou une structuration d'une intention signifiante ; l'inclusion de productions d'apprenants repérées par l'enseignant(e) est souhaitable lorsqu'elles sont correctes ;

- l'enrichissement progressif du matériel initial est envisageable au fur et à mesure des réactions des apprenants;

- la contextualisation « locale » du matériel est importante parce qu'un apprenant sinophone et un apprenant hispanophone n'ont pas le même substrat grammatical, de même qu'un apprenant sinophone sans langue pivot et un apprenant sinophone qui utilise l'anglais comme langue pivot n'auront pas les mêmes références; mais nos dispositifs présentent encore des faiblesses sur ce point ;

- on recourra à un métalangage construit (avec d'éventuelles passerelles avec le métalangage institutionnel);

- ce métalangage doit être avant tout un outil métacognitif parce que son principal objectif est d'être un moyen pour modifier l'activité épilinguistique de l'apprenant;

- il peut prendre diverses formes :

- l'acte de nommer, bien évidemment ;

le recours à des schémas, notamment pour les temps verbaux (cf. Cintas \& Desclés, 1988), la quantification et les relations anaphoriques;

- le recours aux métaphores; par "métaphore», il faut entendre non seulement les métaphores linguistiques mais aussi les métaphores kinésiques et gestuelles (marquage de l'hésitation dans la concession, par exemple);

- l'utilisation de classes paraphrastiques, c'est-à-dire d'ensembles d'énoncés voisins sémantiquement et pragmatiquement (la paraphrase actif / passif en est l'exemple le plus simple) a un double objectif: mettre une formulation donnée en corrélation avec des 
possibilités concurrentes et inciter les apprenants à diversifier leurs productions tout en évaluant la portée d'un tel choix. considérer la grammaire non comme un corps de contraintes mais comme un «jeu d'indices ». Le recours au travail grammatical s'apparente alors au travail de l'enquêteur. La deuxième porte sur les types de médiations à l'œuvre. On peut considérer trois formes de médiation ${ }^{15}$. La médiation sociocognitive est celle qui passe par la négociation des représentations conceptuelles et la schématisation des manières de dire (lors de discussions, de recherches d'inférences, de constitution de matrices, etc.), ce qui converge avec les propositions de Barth (2013, notamment pages 33-53 et 59-64), mais par rapport à un autre public et visant le sens lexical et phrastique. La médiation métalinguistique est celle qui consiste à nommer pour repérer et pour décrire, en cherchant au maximum le partage des façons de nommer et leur intuitivité. La troisième est la médiation épilinguistique qui consiste en l'auto-évaluation par le locuteur de son propre dire et la mise en place d'ajustements pour faire correspondre ce dire à l'intention signifiante et à l'entourage socio-situationnel.

Les apprenants font souvent preuve d'une certaine "cécité » à l'égard de "l'ordre des mots»; cela peut compliquer le processus de l'heuristique (ce qui est évident pour l'enseignant(e) de grammaire ne l'est pas pour l'apprenant).

Une heuristique ne se réalise pas dans un processus de type "automate déterministe ». D'une part, elle a un caractère aléatoire ; d'autre part, elle est constamment guidée "en direct» par un humain, l'enseignant(e). En fait, elle articule des atomisations (par analyse) de séquences linguistiques, des (re)constructions synthétiques de ces séquences et des perceptions holistiques du sens (c'est-à-dire sans capacité à distinguer des composants, alors qu'une synthèse regroupe des composants). De ce fait, il est important de pouvoir agencer des représentations différentes du sens, c'est-à-dire des mises en scène variées du même fait référentiel (Portine, 2013 : 26-27, 45).

\section{En guise de non conclusion}

La grammaire est à la fois medium d'apprentissage d'une langue et discipline (quand les langues deviennent matière d'enseignement, dans quelque dispositif éducatif que ce soit, elles deviennent des objets disciplinaires, Beacco, $2015: 17)$. Nous nous situons ici du point de vue du medium. Notre notion d'heuristique prolonge ce que nous avons repris à la façon dont Christian Lavenne et Jacques Montredon utilisaient C'est le printemps, tout en incluant certains traits des propositions de Besse. Notre notion d'heuristique s'inscrit dans la perspective de centration sur le processus d'apprentissage, y compris du point de vue grammatical (Véronique, 2009). Ce travail est donc loin d'être achevé et notre propos se termine sur un nouveau chantier : la notion de conceptualisation s'est construite sur des bases sociocognitives en ignorant généralement la «contextualisation» de l'acte pédagogique qui devient un sujet d'étude important (cf. Fouillet, Stratilaki-Klein \& Weber, 2015). 


\section{BIBLIOGRAPHIE}

Abry-Deffayet, D. \& Chalaron, M.-L. (2015). La grammaire des premiers temps : B1-B2. Grenoble : PUG.

Aguerre, S. (2010). « Grammaire de l'apprenant et explicitation. Quand apprendre, c'est se construire des représentations, à partir de corpus et d'instruments ». In Galatanu, O. et alii (dir.). Enseigner les structures langagières en FLE. Bruxelles : Peter Lang. pp. 65-75.

Barth, B.-M. (2013). Elève chercheur, enseignant médiateur. Paris : Retz.

Beacco, J.-C. (2015). « La grammaire : Un long fleuve tranquille? ». In Defays, J.-M. et alii. 20 ans de FLES. Faits et gestes de la didactique du Français Langue Etrangère et Seconde de 1995 à 2015. Bruxelles : EME. vol. 1. pp. 15-29.

Benveniste, E. (1964). « Les niveaux de l'analyse linguistique », In Lunt, H.. Proceedings of the $9^{\text {th }}$ International Congress of Linguists. Londres - La Hague: Mouton. pp. 266-275. Repris dans : (1966). Problèmes de linguistique générale, 1. Paris : Gallimard. pp. 119-131.

Besse, H. (1974). « Les exercices de conceptualisation ou la réflexion grammaticale au niveau 2 ». Voix et Images $d u$ CREDIF, $\mathrm{n}^{\circ}$ 2. pp. 39-44.

Besse, H. \& Porquier, R. (1984). Grammaires et didactique des langues. Paris : Hatier-CREDIF [réédition chez Didier en 1991].

Cadet, B. \& Chasseigne, G. (dir.) (2009). Psychologie du jugement et de la décision. Bruxelles : De Boeck.

Cintas, P. \& Desclés, J.-P. (1988). « Signification des temps grammaticaux ». Le français dans le monde, $\mathrm{n}^{\circ}$ 214. pp. 54-59.

Courtillon-Leclerq, J. (1974). « Paraphrase et conceptualisation ». Voix et Images du CREDIF, nº 2. pp. 45-52.

Culioli, A. (1968). « La formalisation en linguistique ». Cahiers pour l'analyse, n 9. pp. 110-117.

Cuq, J.-P. (1996). Une introduction à la didactique de la grammaire en français langue étrangère. Paris : Didier-Hatier.

Fouillet, R., Stratilaki-Klein, S. \& Weber, C. (2015). « Questionner les grammaires du français langue étrangère produites en contexte allophone » In Goes, J. \& Sfar, I. (coord,). La grammaire en FLE/FLS. Quels savoirs pour quels enseignements? Le Français dans le monde. R\&A. $\mathrm{n}^{\circ}$ 57. pp. 38-46.

Gombert, J.-E. (1990). Le développement métalinguistique. Paris : PUF.

Miquel, C. (2013). Grammaire en dialogues : niveau avancé : B2-C1. Paris : Clé international.

Montredon, J. et alii (1982). C'est le Printemps : Livre du professeur. Paris : Clé international.

Montredon, J. (1987). Imparfait et compagnie. Paris : Larousse.

Piaget, J. (1969). Psychologie et pédagogie. Paris : Folio Essais.

Portine, H. (1997). « D’où vient le métalangage ? ». Linx, n 36. pp. 25-39.

Portine, H. (2010). « La notion de "position énonciative" : Sur la question du sujet-apprenant ». Le Français dans le monde, $R \& A, n^{\circ} 48$. pp. 123-134. 
Portine, H. (2013). « Conceptualisation lexicale et grammaticale : Etat des lieux ». In Stratilaki, S. \& Fouillet, R. Éducation aux langues. Contextes et perspectives (Mélanges Jean-Claude Beacco). Paris : Riveneuve. pp. 23-51.

Seguin, J.-P. (1993). L'invention de la phrase au XVIII siècle. Louvain-Paris : Peeters.

Tesnière, L. (1959). Eléments de syntaxe structurale. Paris : Klincksieck.

Trévise, A. (1994). « Représentations métalinguistiques des apprenants, des enseignants et des linguistes : Un défi pour la didactique ». Bulletin suisse de linguistique appliquée, n 59. pp. 171-190.

Trévisiol-okamura, P. \& Kaheraoui, M. (dir.) (2015). Les subordonnées : Corpus, acquisition et didactique. Rennes : PUR.

Véronique, D. (dir.) (2009). L'acquisition de la grammaire du français, langue étrangère. Paris : Didier.

Wilmet, M. (2016). « Postface ». In Coltier, D., Audras, I. \& David, J. Enseigner la grammaire: Contenus linguistiques et enjeux didactiques. Le Français aujourd'hui, $\mathrm{n}^{\circ} 192$. pp. 133-140.

\section{NOTES}

1. Rappelons que «terminologique » signifie " prenant en compte le lien entre terme et concept».

2. Merci à Catherine Bottin, à Caroline Casseville et à Marie-Odile Hidden qui m'ont prêté leurs classes.

3. Une question demeure : l'interlangue est-elle individuelle ou partagée en fonction de la langue maternelle?

4. L'un des relecteurs considère qu'il s'agit là d'un acte de foi et renvoie à l'ouvrage de Markus Gabriel, Ich ist nicht Gehirn (2015) qui critique le neurocentrisme et dont le titre (où Ich renvoie au Moi) est traduit en français de façon un peu racoleuse: Pourquoi je ne suis pas mon cerveau. Précisons que, pour nous, les évolutions de la neuropsychologie (neurosciences nous semble un peu impérialiste) ne sauraient tout expliquer.

5. L'auteur de ces lignes est originaire de Besançon mais vivait à Paris à l'époque de l'élaboration de C'est le Printemps. Les relations amicales conservées avec des collègues du CLAB ont permis de visionner la bande vidéo dont il est question et de discuter avec Christian Lavenne et Jacques Montredon.

6. Piaget (1969: 98-99), commentant Freinet souligne qu'il avait rejoint les deux vérités sans doute les plus centrales de la psychologie des fonctions cognitives: le développement des opérations intellectuelles procède de l'action effective; la coordination générale des actions comporte nécessairement une dimension sociale.

7. http://www.lb.refer.org/fle/cours/cours2_CE/gram/cours2_gr04.htm, site publié avec le sigle de l'AUF. Malheureusement, il est impossible de dater ces propositions (c'est d'ailleurs là un grand problème pour toutes les offres numériques : leur datation).

8. On peut remarquer que l'implication de la notion de concept peut être directe (l'apprenant conceptualise la notion de "pluralité » dans une seconde langue) ou indirecte (un apprenant anglophone mobilise les notions de " possesseur » et de " genre » pour passer de "il regarde son main » à « il regarde sa main »).

9. Tesnière prend l'exemple «La Guerre de Cent-Ans dura 106 ans " face à l'impossible «La Guerre de Cent-Ans durait 106 ans». En donnant la durée de la guerre, on se situe automatiquement du point de vue de sa clôture et non de son déroulement.

10. Nous avons construit ce schéma à des fins pédagogiques à partir des années 2000 en nous appuyant sur les travaux des années 1970 du groupe LNR (Lindsay-Norman-Rumelhart) qui 
opposait conceptually-driven processing (aujourd'hui top-down processing) et data-driven processing (aujourd'hui bottom-up processing).

11. Dans une revue éphémère de l'Ecole normale supérieure de la rue d'Ulm (qui s'est arrêtée au numéro 10).

12. Sur épilinguistique, cf. Gombert (1990), Trévise (1994), Portine (1997).

13. Comme dans les exercices du type : tu regardes une voiture $>$ la voiture que tu regardes.

14. Comme dans les exercices qui demandent de passer de deux phrases à une seule phrase.

15. Nous nous limitons aux aspects qui nous concernent. La notion de médiation a été l'objet d'une véritable explosion de ses emplois : médiation culturelle, scientifique, politique, pénale, etc.

\section{RÉSUMÉS}

L'auteur reprend les différentes notions de grammaire pour mettre en rapport conceptualisation et grammaire mentale. Puis il fait un exposé critique de deux conceptions, celle d'Henri Besse (bien connue) et celle de la méthode C'est le printemps (CLA de Besançon) pour laquelle il apporte un témoignage. Il termine en associant conceptualisation et heuristique.

The author considers the various notions of grammar. His purpose is to match conceptualization and mental grammar. Then, he proposes a critical view of two conceptions: on the one hand, the well-known Henri Besse's conception; on the other hand, the conception found in a method for French as a Foreign Language, C'est le printemps (he provides a personal testimony). He ends by linking conceptualization and heuristics.

\section{INDEX}

Mots-clés : conceptualisation, heuristique, didactique de la grammaire

Keywords : Conceptualization, Heuristics, Grammar Teaching

\section{AUTEUR}

\section{HENRI PORTINE}

Université Bordeaux Montaigne, EA 4595 Telem

Henri Portine a été chargé d'études au BELC de 1976 à 1984 et, dans le même temps, chargé de cours à Paris 7 avant de devenir maître-Assistant puis professeur à Lille 3 et, à partir de 1997, à Bordeaux 3 devenu Bordeaux Montaigne. Il est actuellement professeur émérite. Son doctorat d'état (sous la direction d'Antoine Culioli) portait sur « encore » et la question de la fondation d'une catégorie modale. Ses travaux se répartissent entre linguistique française théorique (temps verbaux, connecteurs rhétoriques, sémantique lexicale) et didactique du français langue étrangère (argumentation et lecture, didactique de la grammaire). Il a été expert pour le MEN (MSTP), l'AERES et quelques autres institutions. 
Site : http://henri-portine.monsite-orange.fr/

Courriel : henri.portine[at]u-bordeaux-montaigne.fr 